\title{
MUSIC EDUCATION IN EARLY CHILDHOOD -AN ANALYSIS
}

\author{
Dr.Kumkum Bharadwaj \\ Department of Drawing \& Painting \\ Govt. M.L.B. Girls P.G.College, Indore
}

The main aim of education is not only giving the knowledge but education is the acquisition of the art of utilization of knowledge. This is an art very difficult to impart . The general purpose of education is the faster growth of individual knowledge in each human being. In human beings projection of individual is only possible by fine arts. Fine arts includes music, dance, architecture, sculpture, painting, literature etc. Music is a performing art. The main aspect of music is Naad. According to Indian views the place of Naad has taken Dhwani. Naad or dhwani is very useful in music. It is the base of music. The main elements of music are -

Swar-In Indian music, there are 7 and in western music "swar" are 8.

Taal- There are 3 types of Taal- Mandra, Madhya, and Drut.

Aaroh- Avroh-Rhythm. (Pitch higher/lower)

Goonj (Echo) - Pratidhwani/Reverberation.

Anunaad-Resonance.

Kaku-Modulation (Scale)

To developing the individual of human being, music is an art, when a child being developing position has effected by Dhwani .It is natural process. Gradually step by step this process is running in human life so that it is important the music education for early childhood.

Early childhood music education is a major segment of children's social, emotional, physical, intellectual and psychological life. Although music and other arts hold their own unique values and procedures, there are specific points that are common to both music and general education of children. For example social interaction, development and physical co-ordination, memory ,psychological, intellectual perception are developments; which are as deeply involved in music learning as they are in the general curriculum. Although general education subjects develop the whole gamut of these skills in the way music education does.

The culturally diverse and international nature of music has ensured the development of a wide range of curriculum implementation method. Singing is a vocally based approach and more creative. These approaches work well in early childhood. Parents link successfully child program, other approaches include musical play, and experimental music which learns by exploration and discovery, whole languages approach linking directly to songs, stories and words. The musical development and behavioral development in early childhood. Musical development of singing voice is also as crucial in teacher development as it is with young children. Since a different part of the cortex is used to sing with than is used to generate speech the development of singing voice helps improve brain development at all ages and informs the 


\section{INTERNATIONAL JOURNAL Of RESEARCH -GRANTHAALAYAH

learning process with a different modality and therefore improves learning itself. The singing voice is natured because it is a valuable tool in a child's early learning experiences. Singing is a major part of a young child's musical life so some skill in vocal training is essential for educators involved in this area. The first five years of a child's musical life is important influence music learning theory? Singing' moving' playing and creating interconnected components in the developmental view that includes input from teacher, carers and parents.

When child entering an early childhood program always come with some musical background, the teacher needs to expose children to a wide variety of music creation, expression and exploration takes place through a variety of modalities as a matter of course so the education needs to utilize as many methods and technologies as they can do further musical development of the child as early as possible in life. Children can then be exposed to varying melodies, rhythm, harmonies, texures and timbers. Young children should be introduced to an electric range of music styles and periods and music of other cultures. The early childhood music program should be more learning based. A balance of teaching styles between group session and child initiated experiences is always recommended as a spontaneous exploration of musical ideas. Group session encourage a valuable singing sound and should share a wide variety of musical games.

These activities provide time for sharing emotional intellectual and physical enjoyment. Through singing and music games children learn how to move sing and play. Singing enhances the skills: beat, pace, tempo, meter, rhythm and rhythmic patterns relative pitch higher/ lower pitch, skip, steps, unison, leaps melodic contour, tonality, head voice, versus speaking voice, structure phrase, repetition ,expressive elements, comparative softer/ louder, dynamics, timbre. Playing instruments should given music making, clapping, stamping, vocalization, slapping thighs etc. Parents, teachers, educators and in general are becoming more aware of the latest research concerning strong relationship between music education in the early years and improved educational outcomes though out their lives. Through the music education improved problems solving skills, reasoning skills, language skills and mathematical abilities increase a short and long term memory. Social awareness enhanced creative intelligence and develops logical brain function, enhanced ability to think and understand.

Here are many challenges of music education, parental and community support, lack of music organization, evaluation of music education policy, base planning and implementation teacher knowledge, understanding skill. We all tried the best in this way the new trend will be in music.

\section{REFERENCES-}

1 Herbert Reade --- "Education through art "page - 3

2 Swantantra Sharma --- "Indian music - a scientific analysis "--- page -8--9--\&-- 21

3 Todays challenge: "Music Education Journal 1989--Andres B'

4 National review of School Music Education -page-- 80 